\title{
COSY-11: an experimental facility for studying meson production in free and quasi-free nucleon-nucleon collisions.
}

\author{
P. Klaja*, H.-H. Adam ${ }^{\dagger}$, A. Budzanowski**, R.Czyżykiewicz ${ }^{\ddagger *}$, \\ D. Grzonka ${ }^{\ddagger}$, M. Janusz ${ }^{*}$, L. Jarczyk*, B. Kamys*, A. Khoukaz ${ }^{\dagger}$, \\ K. Kilian ${ }^{\ddagger}$, P. Moskal ${ }^{*}$, W. Oelert ${ }^{\ddagger}$, C. Piskor-Ignatowicz* ${ }^{*}$ J. Przerwa*, \\ J. Ritman ${ }^{\ddagger}$, T. Rożek ${ }^{\ddagger}$, T. Sefzick ${ }^{\ddagger}$, M. Siemaszko ${ }^{\S}$, J. Smyrski*, \\ A.Täschner ${ }^{\dagger}$, J. Wessels ${ }^{\dagger}$, P. Winter ${ }^{\ddagger}$, M. Wolke ${ }^{\ddagger}$, P. Wüstner ${ }^{\text {Il }}$ and \\ W. Zipper ${ }^{\S}$
}

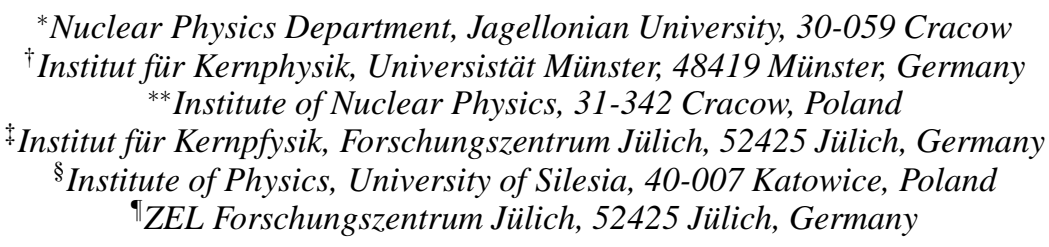

Abstract. The COSY-11 experimental setup is an internal facility installed at the COoler SYnchrotron COSY in Jülich. It allows to investigate meson production in free and quasi-free nucleon-nucleon collisions, eg. $p p \rightarrow$ ppmeson and $p d \rightarrow p_{s p}$ npmeson reactions. Drift chambers and scintillators permit to measure outgoing protons, separated in magnetic field of COSY-11 dipole. Neutrons are registered in the neutron modular detector installed downstream the beam. Recently, the experimental setup has been extended with spectator detector, deuteron drift chamber and polarization monitoring system, and since then meson production can be investigated also as a function of spin and isospin of colliding nucleons.

\section{COSY-11 EXPERIMENTAL SETUP}

The COSY-11 [1, 2, 3] is an internal facility at the COoler SYnchrotron COSY [4, 5] in Jülich, Germany. The recently extended experimental setup is presented in figure 1. It permits to investigate meson and hyperon production in proton-proton and protondeuteron reactions close to the kinematical threshold. A very high experimental precision achieved for the four-momenta determination allows to study the excitation function for the hyperons [6, 7] and mesons [8] creation down to the fraction of $1 \mathrm{MeV}$ above the threshold. In general the reactions are studied by the determination of the four momentum vectors of colliding and outgoing nucleons (in case of the $p p \rightarrow p Y K^{+}$also four momentum vector of the $K^{+}$meson is determined). In front of a normal C-type COSY bending magnet, there is installed a cluster target. Both, hydrogen or deuteron targets [9, 10] can be used.

Due to smaller momenta of positively charged reaction products, they are separated from 


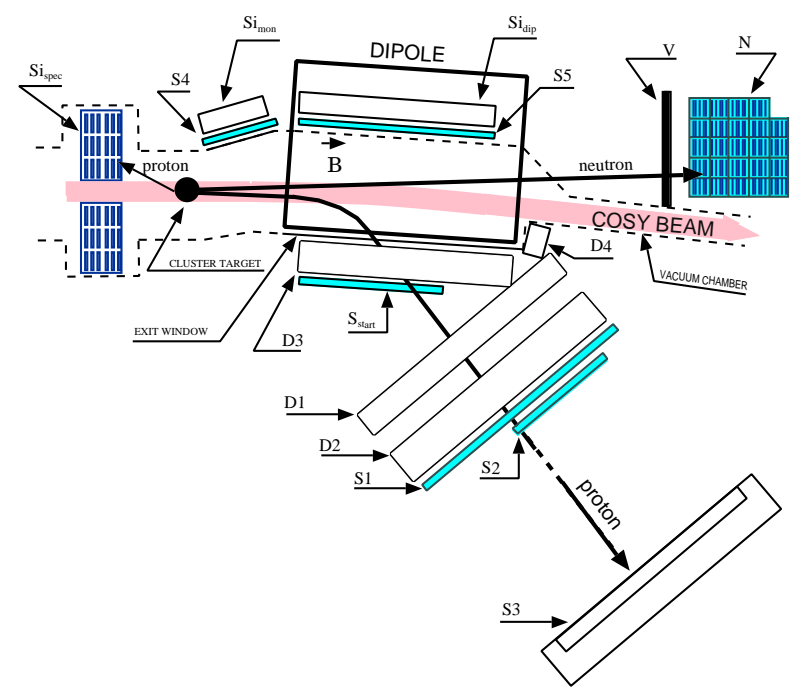

FIGURE 1. Schematic view of COSY-11 detection setup [1]. D1, D2, D3 and D4 denote the drift chambers; S1, S2, S3, S4, S5, $S_{\text {start }}$ and V the scintillation detectors; N the neutron detector and $S i_{m o n}$, $S i_{s p e c}$ and $S i_{d i p}$ silicon strip detectors to detect elastically scattered, spectator protons and negatively charged particles, respectively.

the circulating beam in the magnetic field of the COSY magnet and diverted towards the detection setup. Passing through a specially developed large exit foil of a vacuum chamber, which is mounted inside the dipole gap, the ejectiles reach the detection system operated in normal atmosphere [1].

Negatively charged particles, with their tracks being bent towards the inside of the dipole gap, are detected by an array of silicon detectors $\left(S i_{d i p}\right)$ and an additional scintilation counter (S5), both mounted inside the gap [1].

Neutral reaction ejectiles are detected with the modular neutral particles detector $(\mathrm{N})$ installed downstream the beam [11].

The detection setup has been extended with spectator detector $\left(S i_{\text {spec }}\right)$ [12] and beam polarization monitoring system [13, 14]. Therefore at present, the meson production can be investigated also as a function of spin [15] and isospin [16] of colliding nucleons. The newly installed deuteron drift chamber (D4) [17] permits to study also meson production in $p d \rightarrow p d X$ reactions. The spectator detector is installed inside the vacuum chamber as it is shown in the figure 1. Part of the COSY-11 detection setup used for the registration of elastically scattered protons in the proton-proton free and quasi-free collisions, is shown in figure 1. Trajectories of protons scattered in the forward direction are measured by means of two drift chambers [3] and a scintillation hodoscope (S1), whereas the recoil protons are registered in coincidence with forward ones using a silicon pad detector arrangement $\left(S i_{m o n}\right)$ and scintillation detector (S4) [2]. 


\section{EXPERIMENTAL METHODS}

Identification of registered particles proceeds including various methods. Positively charged ejectiles are identified by independent measurements of their momentum and velocity. For neutrons and gamma quanta velocity vector is obtained. Spectator protons are identified by measurements of their kinetic energy and momentum direction.

For the $p p \rightarrow p p X$ reaction, the collision of protons results in the production of meson. Two ejected protons have smaller momenta and they are separated in the dipole magnetic field from the circulating beam. For this type of reactions, the hardware trigger [18], based on signals from scintillation detectors, was adjusted to register all events with at least two positively charged particles. Tracking back trajectories from drift chambers [3] through the dipole magnetic field to the target point allowed for the determination of the particles momenta. Having momentum and velocity, the latter measured using scintillation detectors, it is possible to identify the mass of the particle [19]. As an example, figure 2 shows the squared mass of two simultaneously detected particles for $p p \rightarrow A^{+} B^{+} X$ reaction (left panel) and $d p \rightarrow A^{+} B^{+} X$ reaction (right panel). Measured reactions can be grouped according to the type of ejectiles. The reaction with two protons, proton and pion, proton and deuteron, pion and deuteron and two pions can be very clearly separeted.
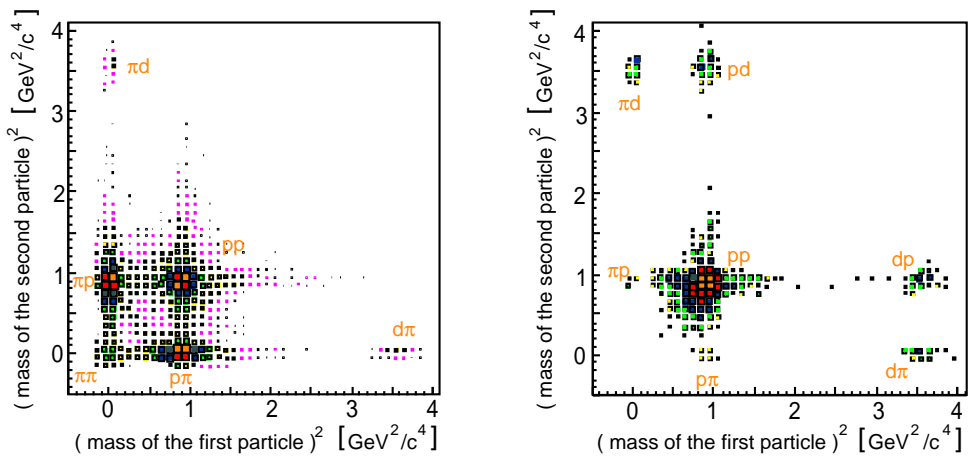

FIGURE 2. Left: Squared masses of two positively charged particles measured in coincidence in the $p p \rightarrow A^{+} B^{+} X$ reaction [20]. Right: Squared masses of two positively charged particles measured in coincidence in the $d p \rightarrow A^{+} B^{+} X$ reaction [21].

The knowledge of the momenta of both protons, for example in the $p p \rightarrow p p X$ reaction, before and after the reaction allows to calculate the mass of an unobserved particle or system of particles created in the reaction. An unobserved meson is identified via the missing mass technique. Figure 3 demonstrates the achieved missing mass resolution for the $p p \rightarrow p p X$ reaction measured close to the $\eta^{\prime}$ meson production threshold, at the COSY-11 detection system, when using a stochastically cooled beam [19]. It is worth noting that the experimental mass resolution is comparable with the natural width of the $\eta^{\prime}$ meson $\left(\Gamma_{\eta^{\prime}}=0.202 \mathrm{MeV}[22]\right)$. 


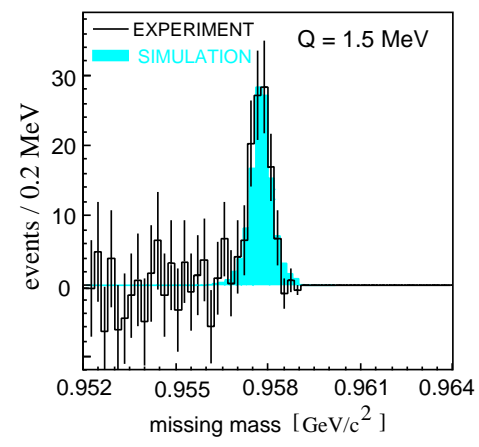

FIGURE 3. Missing mass distribution for the $p p \rightarrow p p X$ reaction measured close to the $\eta^{\prime}$ meson production threshold [23]

\section{ACKNOWLEDGMENTS}

The work has been supported by the European Community - Access to Research Infrastructure action of the Improving Human Potential Programme, by the FFE grants (41266606 and 41266654) from the Research Centre Jülich, by the DAAD Exchange Programme (PPP-Polen), by the Polish State Committe for Scientific Research (grant No. PB1060/P03/2004/26), and by the RII3/CT/2004/506078 - Hadron Physics-Activity -N4:EtaMesonNet.

\section{REFERENCES}

1. S. Brauksiepe et al., Nucl. Instr. and Meth. A 376 (1996) 397.

2. P. Moskal et al., Nucl. Instr. and Meth. A 466 (2001) 448.

3. J. Smyrski et al., Nucl. Instr. and Meth. A 541 (2005) 574.

4. D. Prashun et al., Nucl. Instr. and Meth. A 441 (2000) 167.

5. R. Maier et al., Nucl. Instr. and Meth. A 390 (1997) 1.

6. P. Kowina et al., Eur. Phys. J. A 22 (2004) 293.

7. S. Sewerin et al., Phys. Rev. Lett. 83 (1999) 682.

8. J. Smyrski et al., Phys. Lett. B 474 (2000) 182.

9. H. Dombrowski et al., Nucl. Instr. and Meth. A 386 (1997) 228.

10. A. Khoukaz et al., Eur. Phys. J. D 5 (1999) 275.

11. J. Przerwa, e-Print Archive: hep-ex/0408016 diploma thesis JU (2004).

12. R. Biliger et al., Nucl. Instr. and Meth. A 457 (2001) 64.

13. R. Czyżykiewicz, AIP Conf. Proc. 717 (2004) 858.

14. R. Czyżykiewicz, Schriften des FZ-Jülich, Matter and Materials 21 (2004) 12.

15. P. Winter et al., Phys. Lett. B 544 (2002) 251; Erratum-ibid. B 553 (2003) 339.

16. P. Moskal, AIP Conf. Proc. 717 (2004) 907.

17. C. Piskor-Ignatowicz, P. Moskal, J. Smyrski, Schriften des FZ-Jülich, Matter and Materials 21 (2004) 272.

18. M. Wolke, PhD thesis, University of Bonn (1998); Berichte des FZ-Jülich, Jül (1998), available at http://ikpe1101.ikp.kfa-juelich.de/

19. P. Moskal, e-Print Archive: hep-ph/0408162 habilitation thesis.

20. P. Moskal et al., Phys. Lett. B 474 (2000) 416.

21. J. Przerwa et al., Int. J. Mod. Phys. A 20 (2005) 625.

22. K. Hagiwara et al., Phys. Rev. D 66 (2002) 010001.

23. P. Moskal et al., Phys. Rev. Lett. 80 (1998) 3202. 